\title{
Recent progress in vaccines against foot-and-mouth disease
}

\author{
WIES $Ł A W$ NIEDBALSKI, ANDRZEJ FITZNER, KRZYSZTOF BULENGER
}

Department of Foot and Mouth Disease, National Veterinary Research Institute, Wodna 7, 98-220 Zduńska Wola, Poland

Niedbalski W., Fitzner A., Bulenger K.

Recent progress in vaccines against foot-and-mouth disease

\section{Summary}

Foot-and-mouth disease (FMD) is the most highly contagious disease affecting livestock resulting in a significant adverse economic impact worldwide. Disease outbreaks in previously FMD-free countries are initially controlled by the culling of infected and in-contact animals, restriction of susceptible animal movement, and vaccination with an inactivated whole-virus antigen preparation. With increasing trade, commerce globalization, and people migration, it is likely that more inter-pool viral exchanges and spillovers will occur posing a greater threat to both, endemic and non-endemic regions, mostly due to the limited antigenic coverage of current vaccines. Currently available inactivated FMD vaccines have a number of disadvantages, including incomplete inactivation of the virus, they require multiple vaccination to maintain good levels of immunity and periodic inclusion of new viral strains into the vaccine formulation to cover new FMDV subtypes against which existing vaccines no longer protect and lack differentiation of infected from vaccinated animals (DIVA). The essential aim of DIVA strategy is realization of the so-called "vaccinate-to-live" policy, which is based on the principles that vaccinated animals exposed to FMDV will not transmit the virus. To address the shortcomings of inactivated vaccines, many efforts are currently devoted to developing novel FMD vaccines, including attenuated and marker inactivated vaccines, recombinant protein vaccines, synthetic peptide vaccines and empty capsid vaccines. Novel vaccine platforms offer promising alternatives for effective FMD control. It is likely that in the near future, multiple FMD vaccine approaches will compete for diverse markets, providing fit-for purpose solutions to evolving challenges in preventing and controlling FMD worldwide.

Keywords: foot-and-mouth disease virus (FMDV), inactivated marker vaccines, novel vaccines, progress

Foot-and-mouth disease (FMD) is the most highly contagious disease affecting livestock resulting in significant adverse economic impact worldwide (16). The disease is caused by infection with the foot-and-mouth disease virus (FMDV), the prototypic member of the Aphthovirus genus within the family Picornaviridae. FMDV exists in seven distinct serotypes comprising $\mathrm{O}$, A, C, Asia-1 and SAT 1-3 and multiple subtypes due to the high mutational rate of the virus. The virion compromises a single strand positive sense RNA genome encapsidated in a non-enveloped icosaherdal particle of about 25-30 nm (1). FMDV is translated as a single polyprotein that is posttranslationally cleaved to produce partial and full cleavage products resulting in four structural proteins (VP1 to VP4) and ten nonstructural proteins (NSP), which play different key roles during the viral replication cycle (12).

FMD is endemic in large areas of the continents of Africa and Asia. In South America, recent outbreaks of FMD occurred in July 2017 in Colombia (http://www.
oie.int/wahis_2/public/wahid.php/Diseaseinformation/ WI). Viruses circulating in endemic regions are classified into seven separate endemic pools based on their antigenic and epidemiological properties (Fig. 1). Strict control on the importation of animals and agricultural products allows Europe, North America and Australia to maintain free status without vaccination. The main legislative act of the EU regulating the control of footand-mouth disease is Council Directive 2003/85/EC of 29 September 2003 on Community measures for the control of foot-and-mouth disease. According to this document FMD outbreaks in previously FMDfree countries are initially controlled by the culling of infected and in-contact animals, restriction of the movement of susceptible animals, disinfection of infected premises, and occasionally emergency vaccination with an inactivated whole-virus antigen preparation, as it was the case in 2001 FMD epizootic in the Netherlands (41). However, currently available FMD vaccines confer protection against clinical FMD although they do 


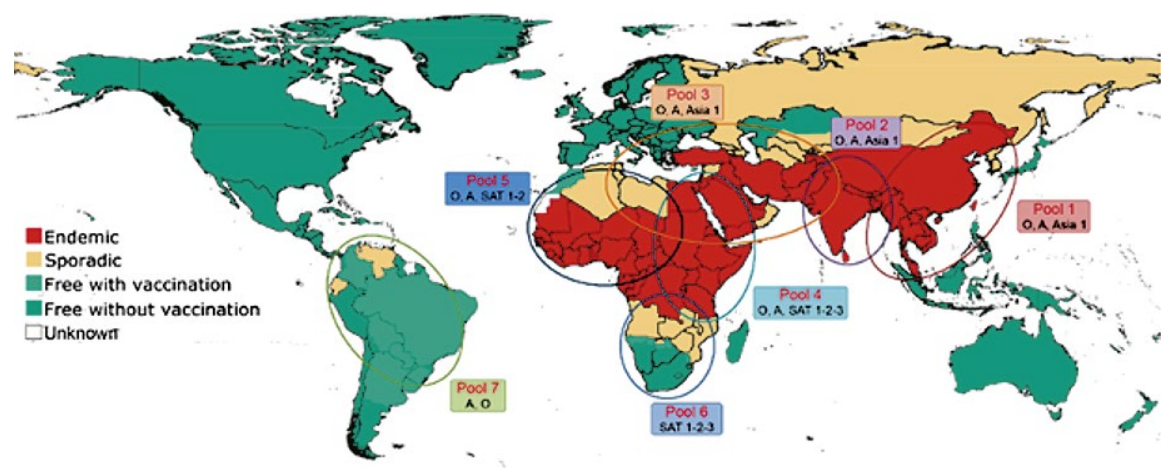

Fig. 1. Distribution of the seven endemic pools of FMDV showing global status of the disease. According to OIE/FAO Reference Laboratory Network for Footand-Mouth Disease (http://www.foot-and-mouth.org/)

not prevent primary infection of the nasopharyngeal mucosa (37), and persistent FMDV infection occurs at a similar prevalence in vaccinated and naive cattle $(37,47)$. Thus vaccination is an efficient measure to prevent clinical FMD and spread of the virus and has proven to be a highly useful strategy in preventing the dissemination of FMD outbreaks, as shown during the 2001 FMD epizootic in Europe when the Netherlands opted to vaccinate all FMD susceptible species, but vaccinated animals were killed to regain FMD-free status and lift trading restrictions more rapidly. FMDfree countries are usually reluctant to use vaccination to control FMD outbreaks because current regulations of the World Organization for Animal Health (OIE) state that countries that slaughter all infected and susceptible in-contact animals, or vaccinate and then slaughter all immunized animals, can regain FMD-free status in three months, while countries that vaccinate and do not slaughter these animals, must wait six months prior to regaining FMD-free status (36). The reasons for this policy clearly reflect the difficulty of unambiguously discriminating infected from vaccinated animals (DIVA) using currently approved diagnostic tests and the fact that properly vaccinated ruminants can still be asymptomatically infected and become carriers (38).

The first inactivated vaccine against FMD was developed around 1937 in Germany by Waldman and coworkers using vesicular fluid obtained from tongues of FMDV infected cattle. However, industrial production of inactivated vaccines did not begin until the 1947 after Frenkel and coworkers at the State Veterinary Research Institute in the Netherlands applied the culture in vitro of tongue epithelium from healthy slaughtered animals for FMDV multiplication (24). Next, in the 1960s the inactivated FMD vaccine production included the growth of FMDV in BHK cell suspension cultures, the introduction of binary ethyleneimine (BEI) for FMDV antigen inactivation, and the use of oil-adjuvant in the 1970s (25). Industrial ultra-filtration and chromatography are used in order to remove unwanted cellular protein contaminants and viral NSP. The need to further purify vaccine antigens arose not only to prevent unwanted allergic reactions to cells proteins in animals after multiple vaccination but also to allow the DIVA strategy during control campaigns (11). Following manufacturing, the inactivated vaccine goes through quality control testing, including identity, sterility, safety, potency, efficacy and contaminant FMDV NSP. Inactivated virus vaccines have been used to effectively control FMD in many developing countries, and such vaccines have helped eliminate the disease from some areas of the world (42). These vaccines usually elicit high levels of neutralizing antibodies and offer efficacious protection against homologous FMDV serotypes (12). However, conventional inactivated FMD vaccines have a number of disadvantages, including incomplete inactivation of the virus, they do not induce broadly reactive long-term protection, require multiple vaccination to maintain good levels of immunity and require periodic inclusion of new viral strains into the vaccine formulation to cover new FMDV subtypes against which existing vaccines no longer protect. It is usually done by the matching of representative field isolates from outbreaks around the world (39). Other important shortcomings of current inactivated vaccines include short shelf life, the need for an adequate cold chain of formulated vaccines, and difficulties of certain FMDV serotypes and subtypes to grow well in cell cultures for vaccine production. Additionally, the used conventional FMD inactivated vaccines do not strictly have DIVA characteristics (10). However, high-quality current FMDV vaccines properly used have been useful in eliminating the disease in many regions of the world. More detailed information about the conventional FMD inactivated vaccines, the history of FMD vaccination and FMD vaccine banks in Europe have been described in our previous publications $(34,35)$.

Because commercially available conventional FMD inactivated vaccines have their specific disadvantages and lack differentiation of infected from vaccinated animals there was necessity for vaccine improvement in order to solve problems of the currently marketed vaccines. The new inactivated whole virus marker vaccines were prepared using attenuated FMDV that grow well in vitro but are harmless to animals. It has been shown that FMD viruses lacking the leader protease coding region $\left(\mathrm{L}^{\mathrm{pro}}\right)$ are attenuated in vivo, and the use of this virus after inactivation was as effective as the wild type inactivated antigen (27). A leaderless infectious clone was used to develop an improved inactivated vaccine (49). This modified vaccine infectious clone is not only deleted in $L^{\text {pro }}$ but also contains nuclease restriction sites that allow for easy capsid swapping to rapidly derive vaccines against emerging FMDV strains. In addition, the new inactivated vaccine has amino acid changes in antigenic sites of the NSPs 3B and 3D that not only make the vaccine fully compatible with existing DIVA 3ABC-ELISA tests, but also provide opportunities for 
further DIVA test development based on protein 3D (49). This leaderless FMDV 3B3D DIVA inactivated vaccine in formulation with adjuvant fully protected cattle against challenge. Another FMDV marker vaccine with deletion of residues 93 to 143 in the NSP 3A has been reported to protect immunized swine against challenge with homologous FMDV. The results indicate that a marker FMDV vaccine can be potentially developed by deleting an immunodominant epitope in NSP 3A (22).

Alternatives of FMDV inactivated vaccines are also the protein/peptide vaccines. These vaccines do not involve infectious virus, are usually stable, can reach 95\% purity, and could be produced in large scale at relatively low costs (45). Individual capsid proteins expressed in E. coli and small linear peptides identified as B and/or T cell epitopes were shown to be protective against FMD in livestock species $(2,52)$. It was found that VP1 isolated from purified virus induced a neutralizing antibody response in swine and protected both swine and cattle against FMDV challenge (2) and these peptides showed enhanced protection in swine (52). Bittle et al. (5) synthesized peptides of the most antigenic site within VP1, the G-H loop, demonstrating that these peptides could induce high levels of neutralizing antibodies in cattle and protection of 2 out of 3 animals. Moreover, in a large scale evaluation of peptide vaccines against FMD, it was found that none of the tested peptides, at several doses and vaccination schedules, afforded protection above $40 \%$. Protection showed limited correlation with serum neutralization activity and lymphoproliferation in response to whole virus (48). Moreover, multiple antigenic peptides or dendrimers (radial or wedge-like branched macromolecules with a peptide core attached to a defined number of epitopes), displaying one copy of a FMDV T-cell epitope branching into several copies of a B-cell epitope, fully protected swine and cattle $(7,46)$. Recent studies using three synthetic peptides vaccines against the $\mathrm{A} / \mathrm{HuBWH} / \mathrm{CHA} / 2009$ strain of FMDV of 59 to 87 amino acids in size, based on immunogenic epitopes in the VP1, 3A, and 3D proteins conferring $60 \%$ protection in cattle after a single immunization (56).

DNA vaccines represent another safer choice for inactivated vaccines since they do not require high containment facilities for production, have a relatively stable shelf life, allow for rapid incorporation of emerging field strain sequences, can incorporate marker genes and can co-express multiple antigenic sites from different serotypes. Immunization with DNA encoding for full length modified FMDV, empty capsids or smaller sub-genomic regions, in some cases co-expressing immunomodulatory genes, have been evaluated in swine and cattle $(3,8,14)$. However, large amounts of DNA and multiple inoculations were needed to induce a relative low FMDV-specific neutralizing antibody response with inconsistent levels of protection against virus challenges. Similar efficacy was observed when
DNA plasmids were used to deliver specific FMDV $\mathrm{B}$ and $\mathrm{T}$ cell epitopes (15). Incorporation of modifications on these epitopes to target antigen presenting cells demonstrated total protection against a challenge with homologous FMDV in those animals efficiently primed for both, humoral and cellular specific responses. A novel DNA vaccine against FMD based on mannosylated chitosan nanoparticles was evaluated in guinea pigs, demonstrating substantial improvement in all the immunological parameters with enhanced protection (31). Another DNA vaccine containing P1 capsid region and NSP 2A, 3C and 3D was administered using an electroporation delivery device protecting $75 \%$ of cattle after a multiple boost (14).

An alternative approach to protein/peptide and DNA FMD vaccines is the development of immunogens that contain the entire repertoire of immunogenic sites present on the intact virus particle, thereby decreasing the possible selection of antigenic variants from the quasispecies. Empty viral capsids, also called virus like particles (VLPs), are virus particles lacking nucleic acid, with DIVA capabilities, naturally produced in infected cells and are as immunogenic as virions. In earlier studies, FMDV VLPs were expressed in recombinant baculovirus-infected cells, in vitro purified, and inoculated into animals (43). Subsequently a number of approaches have been used to deliver the products either as proteins expressed in E. coli (17) or by direct intramuscular inoculation of recombinant vectors including naked DNA (9), poxviruses (5) and human adenovirus (28). To date, the most successful strategy to induce protective responses in animals has been the use of recombinant-replication-defective human adenovirus type 5 coding for FMDV capsids (Ad5-FMD). In initial efficacy studies an Ad5 vector containing the capsid and 3C protease coding regions of the A12 laboratory strain of FMDV protected swine from clinical disease after contact challenge (28). However, although these products did offer some protection, they did not reach the efficacy of the inactivated whole-virus vaccine because only small amounts of antigen were obtained $(4,43)$. More recently, Ad5-FMD vectors containing the capsid from other FMD serotypes and subtypes have been constructed and successfully tested in cattle (18). To enhance the potency and efficacy of the Ad5FMD vector approach and develop a commercially viable FMD vaccine candidate against many circulating FMDV serotypes, a number of strategies have been successfully evaluated. First, inclusion of full-length 2B coding region improved synthesis of FMDV capsid proteins by inducing drastic rearrangement of intracellular membranes involved in replication, and this change enhanced FMDV-specific neutralizing antibody response in swine (40). Furthermore, an Ad5-FMDV vector containing an extra Arg-Gly-Asp (RGD) motif inserted in the HI-loop of adenovirus fiber proteins was evaluated in cattle (29). The obtained results shows that the introduction of RGD motif in the Ad5 fiber enhanced 
T-cell response in cattle against serotype O1Campos, although no significant increase in vaccine efficacy was found. More recently, Fernandez-Sainz et al. (13) evaluated the efficacy of the Ad5-vectored subunit vaccine that delivers FMDV O1 Manisa capsid and capsid processing proteins (Ad5-O1 Manisa) in swine. Swine inoculated with Ad5-O1 Manisa developed an FMDV-specific humoral response as compared to animals inoculated with an empty Ad-5 vector. Vaccinated animals were completely protected against homologous challenge at 7 or 21 days post vaccination. The obtained results indicate that recombinant Ad5-O1 Manisa is an effective, safe and cross-reacting vaccine that could potentially be used preventively and in the situation of outbreaks to control FMDV O Mya-98 in swine (13). In summary, the main advantages of Ad5-FMD vector vaccine are: first, it does not require a high containment facility for production; second, it has intrinsic DIVA capabilities; third, it does not require the adaptation of field strains to vaccine production in cells; fourth, it is genetically stable, However, the Ad5-FMD approach still requires a relatively high dose to achieve protective responses resulting in high cost production, which is problematic for a veterinary vaccine especially in developing countries.

Over the years, a great effort has been focused on production of VLPs in insect cells because of the simplicity of this system. Recently, interest in the use of VLPs has increased, and new results from testing this technology in cattle have been obtained. Recombinant silkworm (Bombyx mori) baculovirus Bm-P12A3C containing the intact $\mathrm{P} 1-\mathrm{P} 2$ and $\mathrm{C} 3$ protease coding regions of FMDV serotype Asia 1 or serotype A were used to immunize cattle and successfully protected all tested animals (23, 30). A novel chimeric VLP-based vaccine for serotypes $\mathrm{O}$ and Asia 1 FMDV was recently reported to induce the broad cross protection (21) but this new approach has not been tested in cattle yet. Xiao et al. (53) have developed a co-expression system $E$. coli, which drove the expression of FMDV Asia 1 capsid proteins (VP0, VP1, and VP3) in tandem by a single plasmid. The co-expressed FMD capsid proteins were produced in a large scale and the chromatographic purified proteins were auto-assembled as VLPs in vitro. Cattle vaccinated with a single dose of the subunit vaccine, comprising in vitro assembled FMDV VLP and an adjuvant, developed FMDV-specific antibody response within a persistent period of 6 months. Moreover, cattle vaccinated with the subunit vaccine showed the high protection potency with the $50 \%$ bovine protective dose $\left(\mathrm{PD}_{50}\right)$ reaching $11.75 \mathrm{PD}_{50}$ per dose. The authors suggested that in vitro assembled recombinant FMDV VLPs produced from E. coli could function as a potent FMDV vaccine candidate against FMDV Asia 1 infection. The vaccinia virus can also be used as a vector to deliver empty capsids (5). The FMDV empty capsid particles were expressed within mammalian cells infected with vaccinia vectors; however, these vectors have not been tested as FMD vaccine in the natural host (20). A fowlpox virus-based recombinant FMD vaccine containing FMDV capsid polypeptide and $3 \mathrm{C}$ coding regions of FMDV serotype $\mathrm{O}$ induced synthesis of specific anti-FMDV antibody and neutralizing antibody, as well as T lymphocyte proliferation response and cytotoxic $\mathrm{T}$ lymphocytes (CTL) activity in mice and guinea pigs vaccinated with such a recombinant vaccine (57). In addition, this fowlpox virus expressing VLPs, partially protected swine against FMDV challenge (26). Furthermore, different variations of the attenuated pseudorabies virus (PRV) vector have been used to express FMDV empty capsids, although complete protection of swine has never been achieved and no testing in cattle was described (55). Recently, a "single cycle" packaged alphavirus self-replicating RNA vector based on Semliki Forest virus (SFV) has been used to express FMDV VLPs in cattle (rSFV-FMDV). However, animals vaccinated with rSFV-FMDV vector were not protected against a homologous challenge unless they were boosted with in vitro purified FMDV VLPs prior to the challenge (19). Expression of VLPs in plants is another very appealing approach since animals could be immunized by feeding with crops expressing empty FMD capsids. Alfalfa, tomato fruit or tobacco have been targeted as FMD VLPs production platforms (51). However, good efficacy in the natural host remains to be evaluated for supporting further development of this approach.

Rapid and long-lasting protection against FMDV infection can also be achieved by using live attenuated vaccines (LAVs). The first generation of LAVs against FMD relies on the conventional empirical attenuation obtained by repeated virus passage in cultured cells. However, the attenuated viruses created in this manner may revert to virulence and be a potential source of infection with FMDV. Advances in molecular virology - creating deleterious gene mutations, altering replication fidelity, and deoptimizing codons - have provided a new way of controlling viral replication and virulence of FMDV. The genetic alteration of FMDV A12 to delete the entire $\mathrm{L}$ proteinase $\left(\mathrm{L}^{\text {pro }}\right)$ coding sequence, leaderless virus (LLV), rendered a virus with reduced pathogenicity and transmissibility in vivo. Experiments on cattle inoculated with live LLV virus showed induction a strong neutralizing antibody response, although there was not protection after challenge with homologous FMDV (27). Bioinformatics analysis of the primary sequence of $L^{\text {pro }}$ revealed a conserved domain called SAP ( SAF-A/B, actinus, and PIAS). Mutation of the SAP domain within the $L^{\text {pro }}$ coding region also confers significant viral attenuation and, remarkably, it fully protects swine against homologous challenge as early as 2 days post vaccination (44). This evidence provided with this attenuated virus has been further supported by a transcriptome analysis using next generation sequencing (NSG) showing differential expression of many transcripts involved in antiviral response (32). In vivo studies with a genetically engineered virus containing 
insertions at the region between the two polyprotein initiation codons (AUG) showed severe attenuation in cattle inoculated by a simulated-natural aerosol route (1). Other studies based on serial passaging of a "wild type" (WT) virulent FMDV strain in suckling rabbits resulted in different mutations in the $\mathrm{L}^{\text {pro }}$ region, including the inter AUG region, that led to a virus attenuated in cattle (54). Moreover, a chimeric FMDV A24 containing $L^{\text {pro }}$ from bovine rhinitis $B$ virus (BRBV), a related Apthovirus, was attenuated in cattle and induced protective immunity against challenge with wild type homologous FMDV (50). More recently, the use of codon pair deoptimization (CPD)-based approach as a novel strategy to develop live-attenuated FMD vaccine has been applied to FMDV. Deoptimization of the P1 coding region of FMDV A12 resulted in severe attenuation in vitro and in vivo with induction of high titers of neutralizing antibodies in swine and mice. Although no challenge experiments have yet been conducted in livestock species, CPD strategy offers a new generation of attenuated strains that - without affecting antigenicity - mimic natural infection (10).

In conclusion, a variety of vaccine strategies are potentially available for immunization of livestock against FMD infection. However, only inactivated vaccines have been produced commercially and used widely to prevent FMD. Due to the disadvantages of inactivated vaccines there is an obvious increased demand for novel vaccines that feature higher potency, safety, improved DIVA capability and most importantly, adequate coverage of circulating and emerging FMD strains. Alternate vaccine technologies described in this paper could address many of the current inactivated vaccine gaps. A summary of the profile of current inactivated vaccines and the novel "ideal" vaccines profile is presented in Table 1. Synthetic biology has facilitated and broadened the applications of FMD vaccines. For example, capsids and emerging strains can now be bench-synthesized and expressed on many platforms, including attenu-

Tab. 1. Profile of currently available inactivated vaccines and desirable novel vaccines against FMD

\begin{tabular}{|l|c|c|}
\hline \multicolumn{1}{|c|}{ Characteristics } & $\begin{array}{c}\text { Current } \\
\text { inactivated } \\
\text { vaccines }\end{array}$ & $\begin{array}{c}\text { Novel } \\
\text { vaccines }\end{array}$ \\
\hline Induction broadly reactive-term protection & no & yes \\
\hline Single vaccination ensure a protective immunity & no & yes \\
\hline Long shelf life (> 2 year) & no & yes \\
\hline Long lasting immunity (>1 year) & no & yes \\
\hline Cross protection across serotypes & no & yes \\
\hline Prevents primary infection & no & yes \\
\hline Prevents carrier state & no & yes \\
\hline Does not require high containment for production & no & yes \\
\hline DIVA strategy & no & yes \\
Thermal stability & no & yes \\
Cost & moderate & low \\
\hline
\end{tabular}

ated FMDV, live/bacterial vectors, DNA and peptide vaccines. One of the most promising technologies that might be adopted in the short term is the use of an attenuated DIVA-marked FMDV strain as the basis for inactivated vaccine production. In case of preventive vaccination in a FMD-free area at risk, the safe and broad protective vaccines that enable DIVA should be used. The essential aim of DIVA strategy is realization of the so-called "vaccinate-to-live" policy, which is based on the principles that vaccinated animals exposed to FMDV will not transmit the virus (33). Emergency response to outbreaks will require fast acting DIVA compatible vaccines with long-term stability of the ready to use product. These fit-for-purpose, rationally designed vaccine strategies and their companion diagnostic tests will need to be developed based on a deep understanding of the functional genomics of FMDV and the mechanisms of FMDV virulence. It is likely that in the near future, multiple FMD vaccine approaches will compete for diverse markets, providing fit-for purpose solutions to evolving challenges in preventing and controlling FMD worldwide. Therefore, studies on the development of new generation vaccines against FMDV should be continued and intensified.

\section{References}

1. Arzt J., Pacheco J. M., Smoliga G. R., Tucker M. T., Bishop E., Pauszek S. J., Hartwig E. J., de los Santos T., Rodriguez L. L.: Foot-and-mouth disease virus virulence in cattle is co-determined by viral replication dynamics and route of infection. Virology 2014, 452-453, 12-22.

2. Bachrach H. L., Moore D. M., McKercher P. D., Polatnick J.: Immune and antibody responses to an isolated capsid protein of foot-and-mouth disease virus. J. Immunol. 1975, 115, 1636-1641.

3. Beard C.,Ward G., Rieder E., Chinsangaram J., Grubman M. J., Mason P. W: Development of DNA vaccines for foot-and-mouth disease, evaluation of vaccines encoding replicating and non-replicating nucleic acids in swine. J. Biotechnol. 1999, 73, 243-249.

4. Belsham G. J., Abrams C. C., King A. M., Roosien J., VlakJ. M.: Myristoylation of foot-and-mouth disease virus capsid protein precursors is independent of other viral proteins and occurs in both mammalian and insect cells. J. Gen. Virol. 1991, 72, 747-751.

5. Berinstein A., Tami C., Taboga O., Smitsaart E., Carrillo E.: Protective immunity against foot-and-mouth disease virus induced by a recombinant vaccinia virus. Vaccine 2000, 18, 2231-2238.

6. Bittle J. L., Houghten R. A., Alexander H., Shinnick T. M., Sutcliffe J. G., Lerner R. A., Rowlands D. J., Brown F.: Protection against foot-and-mouth disease by immunization with a chemically synthesized peptide predicted from the viral nucleotide sequence. Nature 1982, 298, 30-33.

7. Blanco E., Guerra B., de la Torre B. G., Defaus S., Dekker A., Andreu D., Sobrino F: Full protection of swine against foot-and-mouth disease by a bivalent B-cell epitope dendrimer peptide. Antiviral Res. 2016, 129, 74-80.

8. Cedillo-Barron L., Foster-Cuevas M., Belsham G. J., Lefevre F., Parkhouse R. $M$.: Induction of a protective response in swine vaccinated with DNA encoding foot-and-mouth disease virus empty capsid proteins and the 3D RNA polymerase. J. Gen. Virol. 2001, 82, 1713-1724.

9. Chinsangaram J., Beard C., Mason P. W., Zellner M. K., Ward G., Grubman M. J. Antibody response in mice inoculated with DNA expressing foot-and-mouth disease virus capsid proteins. J. Virol. 1998, 72, 4454-4457.

10. Diaz-San Segundo F., Medina G. N., Ramirez-Medina E., Velazquez-Salinas L., Koster M., Grubman M. J., de los Santos T.: Synonymous deoptimization of foot-and-mouth disease virus causes attenuation in while inducing a strong neutralizing antibody response. J. Virol. 2015, 90, 1298-1310.

11. Doel T. R.: FMD vaccines. Virus Res. 2003, 91, 81-99.

12. Domingo E., Baranowski E., Escarmis C., Sobrino F.: Foot-and-mouth disease virus. Comp. Immunol. Microbiol. Infect. Dis. 2002, 25, 297-308

13. Fernandez-Sainz I., Medina G. N., Ramirez-Medina E., Koster M. J., Grubman M. J., de Los Santos T.: Adenovirus - vectored foot-and-mouth disease vaccine confers early and full protection against FMDV O1 Manisa in swine. Virology 2017, 502, 123-132. 
14. Fowler V. L., Bashiruddin J. B., Maree F. F., Mutowembwa P., Bankowski B., Gibson D., Cox S., Knowles N., Barnett P. V.: Foot-and-mouth disease marker vaccine: cattle protection with a partial VP1 G-H loop deleted virus antigen. Vaccine 2011, 29, 8405-8411.

15. Ganges L., Borrego B., Fernandez-Pacheco P., Revilla C., Fernandez-Borges N., Dominguez J., Sobrino F., Rodriguez F.: DNA immunization of pigs with footand-mouth disease virus minigenes: from partial protection to disease exacerbation. Virus Res. 2011, 157, 121-125.

16. Grubman M. J., Baxt B.: Foot-and-mouth disease. Clin. Microbiol. Rev. 2004 $17,465-493$

17. Grubman M. J., Lewis S. A., Morgan D. O.: Protection of swine against footand-mouth disease with viral capsid proteins expressed in heterologous systems. Vaccine 1993, 11, 825-829.

18. Grubman M. J., Moraes M., Schutta C., Barrera J., Neilan J. G., Ettyreddy D. Butman B. T., Brough D. E., Brake D. A.: Adenovirus serotype 5-vectored footand-mouth disease subunit vaccines: the first decade. Future Virol. 2010, 5,14

19. Gullberg M., Lohse L., Botner A., McInerney G. M., Burman A., Jackson T. Polacek C., Belsham G. J.: A prime-boost vaccination strategy in cattle to prevent foot-and-mouth disease using a single-cycle alphavirus vector and empty capsid particles. PLoS One 2017, 12, e0173327, doi. 10.1371/journal.pone.0173327.

20. Gullberg M., Muszyński B., Organtini L. J., Ashley R. E., Hafenstein S. L., Belsham G. J., Polacek C.: Assembly and characterization of foot-and-mouth disease virus empty capsid particles expressed within mammalian cells. J. Gen. Virol. 2013, 94, 1769-1779.

21. Li H., Li Z., Xie Y., Qin X., Qi X., Sun P., Bai X., Ma Y., Zhang Z.: Novel chimeric foot-and-mouth disease virus-like particles harbouring serotype O VP1 protect guinea pigs against challenge. Vet. Microbiol. 2016, 183, 92-96.

22. Li P., Lu Z., Bai X., Li D., Sun P., Bao H., Fu Y., Cao Y., Chen Y., Xie B., Yin H., Liu Z.: Evaluation of a 3A-truncated foot-and-mouth disease virus in pigs for its potential as a marker vaccine. Vet. Res. 2014, 45, 51, doi: 10.1186/1297-971645-51.

23. Li Z., Yi Y., Yin X., Zhang Y., Liu M., Liu H., Li X., Li Y., Zhang Z., Liu J.: Development of foot-and-mouth disease virus serotype A empty capsid subunit vaccine using silkworm (Bombyx mori) pupae. PLoS One 2012, 7, e43849, doi:10.1371/journal.pone.0043849

24. Lombard M., Fussel A. E.: Antigen and vaccine banks: technical requirements and the role of the european antigen bank in emergency foot and mouth disease vaccination. Rev. Sci. Tech. 2007, 26, 117-134.

25. Lombard M., Pastoret P. P., Moulin A. M.: A brief history of vaccines and vaccination. Rev. Sci. Tech. 2007, 26, 29-48.

26. Ma M., Jin N., Shen G., Zhu G., Liu H. J., Zheng M., Lu H., Huo X., Jin M., Yin G., Ma H., Li X., Ji Y., Jin K.: Immune responses of swine inoculated with a recombinant fowlpox virus co-expressing P12A and 3C of FMDV and swine IL-18. Vet. Immunol. Immunopathol. 2008, 121, 1-7.

27. Mason P. W., Piccone M. E., Mckenna T. S., Chinsangram J., Grubman M. J.: Evaluation of a live-attenuated foot-and-mouth disease virus as a vaccine candidate. Virology 1997, 227, 96-102.

28. Mayr G. A., O'Donnell V., Chinsangram J., Mason P. W., Grubman M. J.: Immune responses and protection against foot-and-mouth disease virus (FMDV) challenge in swine vaccinated with adenovirus-FMDV constructs. Vaccine 2001, $19,2152-2162$

29. Medina G. N., Montiel N., Diaz-San Segundo F., Sturza D., Ramirez-Medina E., Grubman M. J., de los Santos T.: Evaluation of a fiber-modified adenovirus vector vaccine against foot-and-mouth disease in cattle. Clin. Vaccine Immunol. 2015, 23, 125-136.

30. Mohana Subramanian B., Madhanmohan M., Sriraman R., Chandrasekhar Reddy R. V., Yuvaraj S., Manikumar K., Rajalakshmi S., Negendrakumar S. B., Rana $S$. K., Srinivasan $V$. A.: Development of foot-and-mouth disease virus (FMDV) serotype O virus-like-particles (VLPs) vaccine and evaluation of its potency. Antyviral Res. 2012, 96, 288-295.

31. Nanda R. K., Hajam I. A., Edao B. M., Ramya K., Rajangam M., Chandra Sekar S., Ganesh K., Bhanuprakash V., Kishore S.: Immunological evaluation of mannosylated chitosan nanoparticles based foot and mouth virus DNA vaccine, pVAC FMDV VP1-OmpA in guinea pigs, Biologicals 2014, 42, 153-159.

32. Ni Z., Yang F., Cao W., Zhang X., Jin Y., Mao R., Du X., Li W., Guo J., Liu X., Zhu Z., Zheng H.: Differential gene expression in porcine SK6 cells infected with wild-type and SAP domain-mutant foot-and-mouth disease virus. Virol. Sin. 2016, 31, 249-257.

33. Niedbalski W., Fitzner A.: DIVA strategy in the eradication of foot-and-mouth disease. Med. Weter. 2017, 73, 23-27.

34. Niedbalski $W$., Kęsy A.: Foot and mouth disease vaccine banks in Europe: present status and future prospects. Med. Weter. 2007, 63, 394-398.

35. Niedbalski W., Wijaszka T., Kęsy A.: Marker vaccines - a new approach to controlling and eradicating foot and mouth disease. Med. Weter. 2003, 59, 279-282.

36. OIE.: Terrestrial Animal Health Code. 2016, Chapter 8.8, Foot and mouth disease. Article 8.8.7., Recovery of free status.
37. Pacheco J. M., Smoliga G. R., O'Donnell V., Brito B. P., Stenfeldt C., Rodriguez L. L., Arzt J.: Persistent foot-and-mouth disease virus infection in the nasopharynx of cattle: tissue-specific distribution and local cytokine expression. PLoS One 2015, 10, e0125698, doi: 10.1371/journal.pone.0125698.

38. Parida S., Cox S. J., Reid S. M., Hamblin P., Barnett P. V., Inoue T., Anderson J., Paton D. J.: The application of new techniques to the improved detection of persistently infected cattle after vaccination and contact exposure to foot-andmouth disease. Vaccine 2005, 23, 5186-5195.

39. Paton D. J., Valarcher J. F., Bergmann I., Matlho O. G., Zakharov V. M., Palma E. L., Thomson G. R.: Selection of foot and mouth disease vaccine strains a review. Rev. Sci. Tech. 2005, 24, 981-993.

40. Pena L., Moraes M. P., Koster M., Burrage T., Pacheco J. M., Segundo F. D. Grubman M. J.: Delivery of foot-and-mouth disease virus empty capsid subunit antigen with non-structural protein $2 \mathrm{~B}$ improves protection of swine. Vaccine 2008, 26, 5689-5699.

41. Pluimers F. H., Akkerman A. M., van der Wal P., Dekker A., Bianchi A.: Lessons from the foot and mouth disease outbreaks in the Netherlands in 2001. Rev. Sci. Tech. 2002, 21, 711-721.

42. Rodriguez L. L., Grubman M. J.: Foot and mouth disease virus vaccines. Vaccine 2009, 27, D90-D94.

43. SaizJ. C., Cairo J., Medina M., Zuidema D., Abrams C., Belsham G. J., Domingo E., Vlak J. M.: Unprocessed foot-and-mouth disease virus capsid precursor displays discontinuous epitopes involved in viral neutralization. J. Virol. 1994, $68,4557-4564$

44. Segundo F. D., Weiss M., Perez-Martin E., Dias C. C., Grubman M. J., Santos T. de los: Inoculation of swine with foot-and-mouth disease SAP-mutant virus induces early protection against disease. J. Virol. 2012, 86, 1316-1327.

45. Shao J. J., Wang J. F., Chang H. Y., Liu J. X.: Immune potential of a novel multiple-epitope vaccine to FMDV type Asia 1 in guinea pigs and sheep. Virol. Sin. 2011, 26, 190-197.

46. Soria I., Quattrocchi V., Langellotti C., Gammella M., Digiacomo S., Garcia de la Torre B., Andreu D., Montoya M., Sobrino F., Blanco E., Zamorano P.: Dendrimeric peptides can confer protection against foot-and-mouth disease virus in cattle. PLos One 2017, 12, e0185184, doi: 10.1371/journal.pone.0185184.

47. Stenfeldt C., Diaz-San Segundo F., de Los Santos T., Rodriguez L. L., Arzt J.: The pathogenesis of foot-and-mouth disease in pigs. Front Vet Sci. 2016, 3, 41, doi: $10.3389 /$ fvets.2016.00041.

48. Taboga O., Tami C., Carrillo E., Nunez J. I., Rodriguez A., Saiz J. C., Blanco E., Valero M. L., Roig X., Camarero J. A., Andreu D., Mateu M. G., Giralt E., Domingo E., Sobrino F., Palma E. L.: A large-scale evaluation of peptide vaccines against foot-and-mouth disease: lack of solid protection in cattle and isolation of escape mutants. J. Virol. 1997, 71, 2606-2614.

49. Uddowla S., Hollister J., Pacheco J. M., Rodriguez L. L., Rieder E.: A safe footand-mouth disease vaccine platform with two negative markers for differentiating infected from vaccinated animals. J. Virol. 2012, 86, 11675-11685.

50. Uddowla S., Pacheco J. M., Larson C., Bishop E., Rodriguez L. L., Rai D. K., Arzt J., Rieder E.: Characterization of a chimeric foot-and-mouth disease virus bearing a bovine rhinitis B virus leader proteinase. Virology 2013, 447, 172-180.

51. Veerapen V. P., van Zyl A. R., Wigdorovitz A., Rybicki E. P., Meyers A. E.: Novel expression of immunogenic foot-and-mouth disease virus-like particles in Nicotiana bethamiana. Virus Res. 2018, 244, 213-217.

52. Wang C. Y., Chang T. Y., Walfield A. M., Ye J., Shen M., Chen S. P., Li M. C., Lin Y. L., Jong M. H., Yang P. C., Chyr N., Kramer E., Brown F.: Effective synthetic peptide vaccine for foot-and-mouth disease in swine. Vaccine 2002, 20, $2603-$ -2610 .

53. Xiao Y., Chen H. Y., Wang Y., Yin B., Lv C., Mo X., Yan H., Xuan Y., Huang Y., Pang $W$., Li X., Yuan Y. A., Tian K.: Large-scale production of foot-and-mouth disease virus (serotype Asia 1) VLP vaccine in Escherichia coli and protection potency evaluation in cattle. BMC Biotechnol. 2016, 16, 56, doi: 10.1186/ s12896-016-0285-6.

54. Xin A., Zhu M., Peng Z., Hu Q., Shi C., Liao D., Wang J., Li H.: Genomic changes in an attenuated ZB strain of foot-and-mouth disease virus serotype Asia 1and comparison with its virulent parental strain. Int. J. Genomics 2014, 978609, doi: $10.1155 / 2014 / 978609$.

55. Zhang K., Huang J., Wang $Q$. ., He Y., Xu Z., Xiang M., Wu B., Chen H.: Recombinant pseudorabies virus expressing P12A and 3C of FMDV can partially protect piglets against FMDV challenge. Res. Vet. Sci. 2011, 91, 90-94.

56. Zhang Z., Pan L., Ding Y., Zhou P., Lv J., Chen H., Fang Y., Liu X., Chang H., Zhang J., Shao J., Lin T., Zhao F., Zhang Y., Wang Y.: Efficacy of synthetic peptide candidate vaccines against serotype A foot-and-mouth disease virus in cattle. Appl. Microbiol. Biotechnol. 2015, 99, 1389-1398.

57. Zheng M., Jin N., Zhang H., Jin M., Lu H., Ma M., Li C., Yin G., Wang R., Liu Q. Construction and immunogenicity of a recombinant fowlpox virus containing the capsid and $3 \mathrm{C}$ protease coding regions of foot-and-mouth disease virus. J. Virol. Methods 2006, 136, 230-237.

Corresponding author: Wiesław Niedbalski, DVM, PhD hab., assoc. prof., Wodna 7, 98-220 Zduńska Wola, Poland; e-mail: wieslaw.niedbalski (a)piwzp.pl 